\title{
Anesthesia Management of a Suspected Case of Thyrotoxicosis in a Young Adult Male Undergoing Humerus Surgery
}

\author{
${ }^{1}$ Apeksha A Gala, ${ }^{2}$ Niyati Arora, ${ }^{3}$ Harpreet K Madan, ${ }^{4}$ Naina Dalvi, ${ }^{5}$ Sunil K Gvalani
}

\begin{abstract}
Disease of the thyroid gland in the general population is well defined. Hyperthyroidism is characterized by increased thyroid hormone synthesis and secretion from the thyroid gland, whereas thyrotoxicosis refers to the clinical syndrome of excess circulating thyroid hormones, irrespective of the source. The signs and symptoms of hyperthyroidism if subtle, can be missed during the preoperative period. This case report highlights the importance of vigilant monitoring to diagnose and manage cases of thyrotoxicosis in the intraoperative period.
\end{abstract}

Keywords: Dexamethasone, Intraoperative thyrotoxicosis, Postoperative monitoring, Thyroiditis, Young male.

How to cite this article: Gala AA, Arora N, Madan HK, Dalvi N, Gvalani SK. Anesthesia Management of a Suspected Case of Thyrotoxicosis in a Young Adult Male Undergoing Humerus Surgery. Res Inno in Anesth 2018;3(2):60-62.

Source of support: Nil

Conflict of interest: None

\section{INTRODUCTION}

Disease of the thyroid gland in the general population is well defined. The signs and symptoms of hyperthyroidism need good clinical suspicion and should to be treated preoperatively. Hyperthyroidism is characterized by increased thyroid hormone synthesis and secretion from the thyroid gland, whereas thyrotoxicosis refers to the clinical syndrome of excess circulating thyroid hormones, irrespective of the source. ${ }^{1}$ The difference between the two can be made out only after investigations. Often, the patients present with subtle signs and symptoms, which are overlooked in the preoperative evaluation. In this case

${ }^{1}$ Assistant Professor, ${ }^{2}$ Registrar, ${ }^{3}$ Associate Professor, ${ }^{4}$ Additional Professor, ${ }^{5}$ Professor and HOD

1,2,5 Department of Anesthesiology, HBT Medical College and RN Cooper Hospital, Mumbai, Maharashtra, India

${ }^{3,4}$ Department of Anesthesiology, RN Cooper Hospital, Mumbai, Maharashtra, India

Corresponding Author: Niyati Arora, Registrar, Department of Anesthesiology, HBT Medical College and RN Cooper Hospital, Mumbai, Maharashtra, India, e-mail: drnitiarora@gmail.com report, we intend to emphasize the significance of intraoperative vigilance for the diagnosis of thyrotoxicosis.

\section{CASE REPORT}

A 25-year-old male patient, weighing $60 \mathrm{~kg}$ was posted for lefthumerus implant removal with replating in view of implant failure. Preanesthesia workup did not reveal any significant history. The patient had undergone humerus plating under general anesthesia 6 months back, with no intraoperative or postoperative complications. However, the anaesthesia details were not available with the patient. On preoperative examination, the patient had a pulse of $110 /$ minute, regular. His blood pressure was $130 / 80 \mathrm{~mm}$ of $\mathrm{Hg}$. All other routine investigations were within normal limits. A preoperative ECG was not done according to National Institute for Health and Care Excellence (NICE) guidelines. He did not give any history of significant weight loss or palpitations. However, unusual prominence of eyeballs was noted on examination, which was explained by the parents to be hereditary. On the day of surgery, a written, informed and valid consent was taken. Monitoring included $\mathrm{SPO}_{2}$, ECG, noninvasive blood pressure and end-tidal $\mathrm{CO}_{2}$. His pulse rate was $110 / \mathrm{min}$, regular and BP was $140 / 90 \mathrm{~mm}$ of $\mathrm{Hg}$. Patient was slightly anxious and hence was premedicated with inj midazolam $1.2 \mathrm{mg}$ and injection fentanyl $120 \mathrm{mcg}$. A left brachial plexus block was performed using the interscalene approach with the help of a peripheral nerve stimulator. After the appropriate response, $6 \mathrm{~mL}$ of $2 \%$ lignocaine with $9 \mathrm{~mL}$ of $0.5 \%$ bupivacaine was injected after confirming negative aspiration of blood and CSF. General anesthesia was given as surgery was to be performed in the left lateral position, and patient continued to be anxious. The patient was induced with $120 \mathrm{mg}$ of propofol and after confirmation of ventilation; inj vecuronium $6 \mathrm{mg}$ was given. Patient was ventilated with $\mathrm{O}_{2}+\mathrm{N}_{2} \mathrm{O}$ with sevoflurane for $3 \mathrm{~min}$, and with $100 \% \mathrm{O}_{2}$ for 1 minute thereafter. Endotracheal intubation was performed with a PVC cuffed endotracheal tube no 8 . His pulse rate increased to $140-150 /$ minute, regular, and his BP was 150-160 systolic and 90-100 diastolic. The patient did not show any response to intravenous dexmedetomidine infusion. A decision to start beta blocker (metoprolol) was taken, after patients pulse rate and blood 
pressure continued to remain high. After an initial bolus of $2 \mathrm{mg}$ of inj metoprolol, his pulse rate came down to $120 /$ minute, and BP reduced to $140 / 90 \mathrm{~mm}$ of $\mathrm{Hg}$. However, his tachycardia was persistent and remained in the range of 120-150/minute. The patient also needed a top-up dose of relaxant every 15 minutes. Intravenous dexamethasone was given in view of suspicion of thyrotoxicosis. However, definitive treatment of antithyroid medications was not considered as the cause of thyrotoxicosis was not established. A decision to abandon the procedure was taken. His implant was removed and the rest of the procedure was abandoned. Patient was adequately reversed with inj neostigmine and ing glycopyrrolate and extubated uneventfully. Patient was vigilantly monitored postoperatively for 48 hours in SICU. A postoperative 12 lead ECG, 2DEcho and thyroid function tests was advised. ${ }^{2}$ DEcho was within normal limits. However, a postoperative ECG showed $\mathrm{T}$ wave inversion in leads $\mathrm{V}_{2}$ and $\mathrm{V}_{3}$. Thyroid function test was suggestive of hyperthyroidism with $\mathrm{T}_{3}$ : $378.40 \mathrm{ng} / \mathrm{mL} \mathrm{T}_{4}: 22.16$ microgram/dL, TSH: $<0.005 \mathrm{uIU} /$ $\mathrm{mL}$ (Normal values being $\mathrm{T}_{3}: 84.63-201.8 \mathrm{ng} / \mathrm{mL} \mathrm{T}_{4}: 5.13$ 14.06 microgram/mL, TSH: 0.27-5.50 uIU/mL). Patient was started on tablet propranolol $20 \mathrm{mg}$ twice a day. An ultrasound of his neck was done which was suggestive of thyroiditis and hence patient was not started on antithyroid drugs (Fig. 1).

\section{DISCUSSION}

Thyrotoxicosis affects approximately $2 \%$ of women and $0.2 \%$ of men in the general population. ${ }^{2}$ The optimization of preexisting thyroid conditions in patients undergoing surgery is important for perioperative considerations. ${ }^{3}$ As there are no routine guidelines for screening of thyroid disorders, the preoperative testing is done based on the previous history of thyroid disease and clinical suspicion. Symptoms like unexplained weight loss, palpitations, tremors, change in skin, hair or eyes,

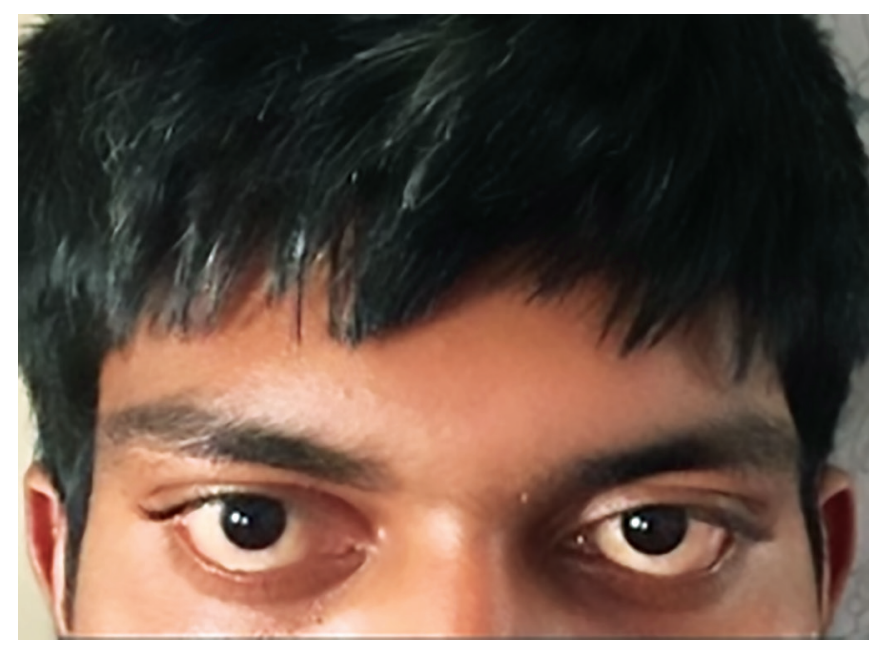

Fig. 1: On preoperative examination; note the prominence of eyeballs or altered bowel habits, are the indicators for a preoperative thyroid function tests. Our patient did not have any of the above-mentioned signs or symptoms, so he was not evaluated further. There are several causes of thyrotoxicosis, including Graves disease, toxic multinodular goiter, toxic adenoma, thyroiditis, and iatrogenic causes. Accurate diagnosis is essential for patients with thyrotoxicosis since its treatment varies according to the cause. For example, in cases of thyrotoxicosis caused by thyroiditis or iatrogenic causes, the administration of antithyroid drugs is contraindicated because in these cases synthesis of thyroid hormones is not increased. ${ }^{4}$ Thyroiditis is generally associated with thyroid lymphocytic infiltration and positive thyroid antibodies. Antithyroid drugs are ineffective and contraindicated due to the deteriorating effects of thyrotoxicosis. ${ }^{5}$ Beta-blocker, steroids, aspirin, and nonsteroidal anti-inflammatory drugs comprise alternative treatment options in thyroiditis. Manifestations of abnormally elevated thyroid hormone levels seen in thyrotoxicosis are numerous and pervade multiple organ systems. The most salient features are the cardiovascular ones, namely, the positive inotropic and chronotropic effects of thyroid hormone on the heart, the vasodilation and decrease in systemic vascular resistance, and the consequent increase in sodium and water retention mediated by the renin-angiotensin-aldosterone system, all of which culminate in an increase in cardiac output by $50-300 \% .^{6}$ The cardiovascular effects of thyrotoxicosis, including atrial fibrillation, congestive cardiac failure, and ischemic heart disease, are the most important conditions requiring monitoring by the anesthesiologist. ${ }^{7}$ Atrial fibrillation occurs in $10-15 \%$ of patients with overt hyperthyroidism and in a similar percentage of those with subclinical hyperthyroidism, i.e., decreased TSH with normal thyroxine and triiodothyronine. ${ }^{8}$ The prevalence of atrial fibrillation increases with age. ${ }^{9}$ Our patient did not show any arrhythmias preoperatively or in the intraoperative period. There are no published studies evaluating the perioperative outcomes of hyperthyroid patients compared with euthyroid patients. Because of the risk of precipitating thyroid storm, a scenario characterized by tachycardia, confusion, fever, gastrointestinal complaints, and potentially leading to cardiovascular collapse, elective surgeries should always be postponed in patients with overt hyperthyroidism. In those with mild or subclinical disease, preoperative $\beta$-blockade is considered sufficient. We suspected thyrotoxicosis intraoperatively on the grounds of patients' tachycardia and blood pressure, not responding to dexmedetomidine infusion, but responding to beta blockers, a patient requiring frequent doses of muscle relaxant, and, a retrospective analysis of the patients' exophthalmos. For the prevention of hypertension and thyroid storm and maintenance 


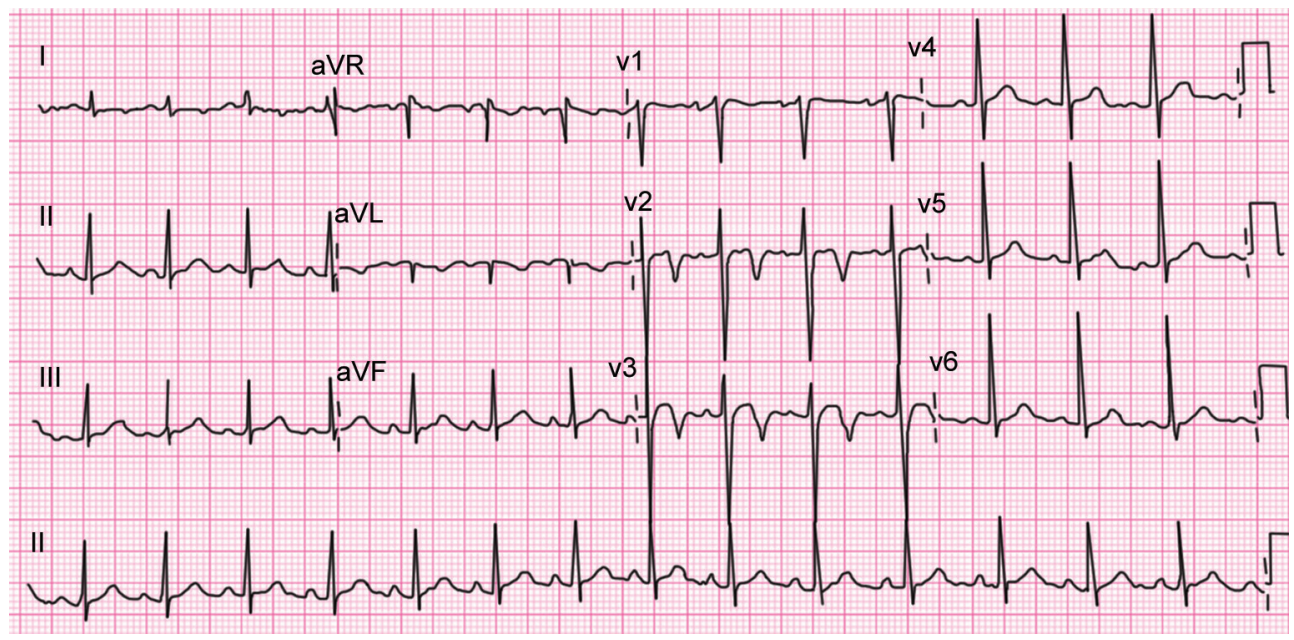

Graph 1: Postoperative 12 lead ECG of the patient

of hemodynamic stability, intravenous metoprolol was administered intermittently. Injection dexamethasone 8 $\mathrm{mg}$ iv bolus followed by $2 \mathrm{mg}$ iv 6 hourly can be given in a patient of thyrotoxicosis (Graph 1$).{ }^{10}$

\section{CONCLUSION}

We conclude that though there is a documented skewed incidence of thyroid disorders in females compared to males, a possibility of hyperthyroidism should be ruled out on the basis of subtle signs.

A high index of suspicion and vigilant monitoring are of utmost importance. Early diagnosis and treatment and deferring surgery in such a patient may prevent overt thyroid storm.

Patients should be given ICU care and monitored postoperatively for at least 48 hours as there is a high risk of cardiac complications.Necessary investigation for accurate diagnosis and its treatment of thyroid disorders should be initiated.

\section{REFERENCES}

1. Kravets I. Hyperthyroidism: Dagnosis and treatment. Am Fam Physician 2016;93(5):363-370.

2. Franklyn J. Thyrotoxicosis. Prescrib J 1999;39:1-8

3. Palace MR. Perioperative management of thyroid dysfunction: Health Serv Insights 2017;10:1178632916689677.

4. Tay S, Khoo E, Tancharoen C, et al. Beta-blockers and the thyrotoxic patient for thyroid and non-thyroid surgery: a clinical review. OA Anaesthetics 2013;1(1):1-6.

5. Franklyn JA, Boelaert K. Thyrotoxicosis. The Lancet 2012;379(9821):1155-1166.

6. Kohl BA, Schwartz S. Surgery in the patient with endocrine dysfunction. Med Clin North Am 2009;93:1031-1047.

7. Donangelo I, Braunstein GD. Update on subclinical hyperthyroidism. American Family Physician 2011;83(8):933-938.

8. Sawin CT, Geller A, Wolf P, et al. Low serum thyrotropin concentrations as a risk factor for atrial fibrillation in older persons. N Engl J Med 1994;331:1249-1252.

9. Auer J, Scheibner P, Mische T, et al. Subclinical hyperthyroidism as a risk factor for atrial fibrillation. Am Heart J 2001;142:838-842.

10. Carroll R, Matfin G. Endocrine and Metabolic Emergencies: Thyroid storm. Ther Adv Endocrinol Metab 2010;1(3):139-145. 